\title{
Worldline Instantons II: The Fluctuation Prefactor
}

\author{
Gerald V. Dunne and Qing-hai Wang \\ Department of Physics, University of Connecticut, Storrs, CT 06269-3046, USA
}

Holger Gies

Institut für Theoretische Physik, Philosophenweg 16, 69120 Heidelberg, Germany

\author{
Christian Schubert \\ Instituto de Física y Matemáticas, Universidad Michoacana de San Nicolás de Hidalgo, \\ Apdo. Postal 2-82, C.P. 58040, Morelia, Michoacán, México
}

In a previous paper [1], it was shown that the worldline expression for the nonperturbative imaginary part of the QED effective action can be approximated by the contribution of a special closed classical path in Euclidean spacetime, known as a worldline instanton. Here we extend this formalism to compute also the prefactor arising from quantum fluctuations about this classical closed path. We present a direct numerical approach for determining this prefactor, and we find a simple explicit formula for the prefactor in the cases where the inhomogeneous electric field is a function of just one spacetime coordinate. We find excellent agreement between our semiclassical approximation, conventional WKB, and recent numerical results using numerical worldline loops.

PACS numbers: 11.27.+d, 12.20.Ds 


\section{INTRODUCTION}

This paper builds on an earlier paper [1], which presented a new way to compute nonperturbative particle production rates in inhomogeneous electromagnetic fields using a semiclassical approximation to Feynman's worldline path integral formulation of quantum electrodynamics [2]. Here we extend this analysis to compute also the subleading prefactor to the particle production rate, by computing the quantum fluctuations about the semiclassical worldline instanton path. The worldline formulation of quantum field theory provides a novel and powerful computational approach to both perturbative and nonperturbative phenomena. Although conceptually as old as relativistic quantum field theory itself, its usefulness for state-of-the-art calculations has been appreciated only in recent years, and largely through its affinity with string methods [3, 4]. String theory methods have been adapted to quantum field theory, which has inspired many further developments [5, 6, 7, 8, 9, 10, 11, 12, 13, 14] beyond Feynman's original proposal (see [15] for a review). In particular, the worldline approach provides an efficient way to compute effective actions in quantum electrodynamics (QED) [16, 17] and quantum chromodynamics (QCD) [18]. These effective actions are the generating functionals for scattering amplitudes, and also contain nonperturbative information, for example concerning particle production. This latter aspect is the subject of this current paper.

The nonperturbative phenomenon of vacuum pair production [19, 20] has applications in many fields of physics [21, 22, 23, 24, 25, 26, 27]. It is a prominent example of a wider class of quantum-induced nonlinear electromagnetic effects 28] which can become accessible in current and future strong-field experiments [29, 30, 31]. As is well-known, the QED pair creation by an external field can be concisely described in terms of the imaginary part of the effective Lagrangian. Affleck et al [32] studied pair production in a constant electric field in scalar QED by applying instanton techniques to the worldline path integral. This semiclassical worldline approach was generalized in [1] to the case of inhomogenous background electric fields. The dominant nonperturbative exponential factor of the imaginary part was computed using special semiclassical worldline loops called worldline instantons. Worldline instantons embody the worldline formulation of the conventional field theoretic WKB computations of Brezin and Itzykson [33] and Popov et al 34], which were in turn motivated by the pioneering ionization studies of Keldysh [35]. Kim and Page [36] have 
found an elegant formulation of pair production in the WKB approach using the language of quantum mechanical instantons, and these results are also complementary to our worldline instanton approach. However, these quantum mechanical instantons are not the same as our "worldline instantons", which are instantons in the proper-time, rather than in the imaginary time of quantum mechanical tunneling computations. Very recently, numerical Monte Carlo techniques have been developed for the calculation of worldline path integrals [37, 38], and applied to both perturbative and nonperturbative QED processes [39, 40]. In this paper we find excellent agreement between these numerical results and our semiclassical approximation.

In Section \we discuss the general idea of worldline instantons as a semiclassical approximation to the nonperturbative part of the worldline effective action. In Section [II] we present a more explicit result for the situation of inhomogeneous electric fields that are just functions of time. Section $1 \mathrm{~V}$ shows how to extend this to the spatially inhomogeneous case. We conclude with a summary and an outline of possible future work in Section $\nabla$

\section{SEMICLASSICAL APPROXIMATION TO THE PATH IN- TEGRAL}

The Euclidean one-loop effective action for a scalar charged particle (of charge $e$ and mass $m)$ in a QED gauge background $A_{\mu}$ is given by the worldline path integral expression [15]

$$
\Gamma_{\text {Eucl }}[A]=-\int_{0}^{\infty} \frac{d T}{T} \mathrm{e}^{-m^{2} T} \int_{x(T)=x(0)} \mathcal{D} x \exp \left[-\int_{0}^{T} d \tau\left(\frac{\dot{x}^{2}}{4}+i e A \cdot \dot{x}\right)\right] .
$$

Here the functional integral $\int \mathcal{D} x$ is over all closed Euclidean spacetime paths $x^{\mu}(\tau)$ which are periodic in the proper-time parameter $\tau$, with period $T$. We use the path integral normalization conventions of [15]. The effective action $\Gamma_{\text {Eucl }}[A]$ is a functional of the classical background field $A_{\mu}(x)$, which is a given function of the space-time coordinates.

If $A_{\mu}$ corresponds to a Minkowskian electric field, then the one-loop Minkowski effective action has a non-perturbative imaginary part associated with the pair production from vacuum. This physical interpretation follows from the fact that the Minkowski effective action is related to the vacuum persistence amplitude as

$$
\langle 0 \mid 0\rangle=e^{i \Gamma_{\mathrm{Mink}}}
$$


An imaginary part of $\Gamma_{\text {Mink }}$ is therefore identified with vacuum non-persistence through pair production, such that

$$
P_{\text {production }}=1-e^{-2 \operatorname{Im} \Gamma_{\text {Mink }}} \approx 2 \operatorname{Im} \Gamma_{\text {Mink }}
$$

For example, if the background electric field is constant and of magnitude $E$, the leading weak-field expression for this imaginary part is

$$
\operatorname{Im} \Gamma_{\text {Mink }} \sim V_{4}^{\text {Mink }} \frac{e^{2} E^{2}}{16 \pi^{3}} \exp \left[-\frac{m^{2} \pi}{e E}\right],
$$

where $V_{4}^{\text {Mink }}$ is the physical (Minkowski) spacetime volume factor.

In 32] it was shown how to compute this leading contribution to the imaginary part of $\Gamma_{\text {Mink }}$ in the constant field case, by using circular semiclassical paths $x_{\mu}^{\mathrm{cl}}(\tau)$ to approximate the path integral in (2.1) for the Euclidean effective action. In [1] this idea was generalized to inhomogeneous electric fields, and the nonperturbative exponential factors were computed in terms of special semiclassical paths called worldline instantons. These worldline instantons reduce to the circular paths of Affleck et al in the limit of a homogeneous background. In this paper we show how to compute also the prefactor to the exponential factors in the inhomogeneous case, by computing the quantum fluctuations about the worldline instantons.

In [1, 32] the proper-time $T$ integral in (2.1) was done first, followed by the quantum mechanical functional integral, each being evaluated by a steepest descents approximation. However, for inhomogeneous background fields this leads to a nonlocal fluctuation problem for the functional integral, which makes the prefactor more difficult to compute. So, here we choose instead to evaluate the integrals in (2.1) in the opposite order. That is, we first make a semiclassical approximation to the quantum mechanical path integral in (2.1), for any $T$, and then evaluate the $T$ integral by steepest descents. It turns out that the form of the worldline instantons remains the same for whichever order of evaluating the integrals, so the results of [1] form the basis of the computation. The basic idea is that since the worldline formulation is essentially a first-quantized approach, we can use results from the study of semiclassical approximations to quantum mechanical path integrals [41, 42].

Consider the quantum mechanical path integral in (2.1), with action $S[x]=\int_{0}^{T} d t L$ for the Lagrangian

$$
L(x, \dot{x})=\frac{\dot{x}^{2}}{4}+i e A \cdot \dot{x}
$$


The Euclidean classical Euler-Lagrange equations are

$$
\ddot{x}_{\mu}=2 i e F_{\mu \nu}(x) \dot{x}_{\nu},
$$

where $F_{\mu \nu}=\partial_{\mu} A_{\nu}-\partial_{\nu} A_{\mu}$ is the background field strength. Note that it follows immediately that for a classical solution $\dot{x}^{2}$ is constant:

$$
\left(\dot{x}^{\mathrm{cl}}\right)^{2}=a^{2} .
$$

Worldline instantons are periodic solutions to (2.6), and several classes of explicit solutions were found in [1]. Furthermore, it was shown in [1] that these classical worldline instantons straightforwardly determine the nonperturbative exponential factor in $\operatorname{Im} \Gamma_{\text {Mink }}$, for inhomogeneous background electric fields.

To compute the prefactor contribution, we need to compute the fluctuations about a worldline instanton. To do this, we need to specify precisely how we sum over all closed loops. There are two standard approaches [6, 15, 43]. The first, which we choose to follow, is to fix a point on each loop and then allow fluctuations about this loop such that the fluctuations vanish at the fixed point. The location of the fixed point is then integrated over. An alternative approach is to consider periodic fluctuations, with the center-of-mass of the loop being kept fixed, and then integrated over at the end. These two approaches are known to give equivalent results after integrating over the loop position [44]. We have further verified that these two approaches also give the same results using worldline instantons, but we found the first approach to be somewhat simpler to implement. Thus we write (2.1) more explicitly as

$$
\Gamma_{\text {Eucl }}[A]=-\int_{0}^{\infty} \frac{d T}{T} \mathrm{e}^{-m^{2} T} \int d^{4} x^{(0)} \int_{x(T)=x(0)=x^{(0)}} \mathcal{D} x \exp \left[-\int_{0}^{T} d \tau\left(\frac{\dot{x}^{2}}{4}+i e A \cdot \dot{x}\right)\right] .
$$

We expand all paths in the functional integral as

$$
\begin{aligned}
& x_{\mu}(\tau)=x_{\mu}^{\mathrm{cl}}(\tau)+\eta_{\mu}(\tau), \\
& \eta_{\mu}(0)=\eta_{\mu}(T)=0
\end{aligned}
$$

The first order expansion of the action vanishes by virtue of the Euler-Lagrange equations, and the quadratic term defines the so-called "secondary action" [41, 45]:

$$
\delta^{2} S[\eta]=\int_{0}^{T} d \tau \eta_{\mu} \Lambda_{\mu \nu} \eta_{\nu},
$$


where the fluctuation operator $\Lambda_{\mu \nu}$ has the following general form

$$
\Lambda_{\mu \nu} \equiv-\frac{1}{2} \delta_{\mu \nu} \frac{d^{2}}{d \tau^{2}}-\frac{d}{d \tau} Q_{\nu \mu}+Q_{\mu \nu} \frac{d}{d \tau}+R_{\mu \nu}
$$

with

$$
\begin{aligned}
Q_{\mu \nu} & \equiv \frac{\partial^{2} L}{\partial x_{\mu} \partial \dot{x}_{\nu}} \\
R_{\mu \nu} & \equiv \frac{\partial^{2} L}{\partial x_{\mu} \partial x_{\nu}}
\end{aligned}
$$

The equations of motion for the fluctuations are known as the Jacobi equations [45]:

$$
\Lambda_{\mu \nu} \eta_{\nu}=0
$$

The semiclassical approximation to quantum mechanical path integrals [41, 42] leads to the following simple result:

$$
\int_{x(T)=x(0)=x^{(0)}} \mathcal{D} x \exp \left[-\int_{0}^{T} d \tau\left(\frac{\dot{x}^{2}}{4}+i e A \cdot \dot{x}\right)\right] \approx \frac{e^{i \theta} e^{-S\left[x^{\mathrm{cl}}\right](T)}}{(4 \pi T)^{2}} \sqrt{\frac{\left|\operatorname{det}\left[\eta_{\mu, \text { free }}^{(\nu)}(T)\right]\right|}{\left|\operatorname{det}\left[\eta_{\mu}^{(\nu)}(T)\right]\right|} .}
$$

The important result from [41, 42] is that the determinant $\operatorname{det}\left[\eta_{\mu}^{(\nu)}(T)\right]$ is simply a finite dimensional $4 \times 4$ determinant, formed from solutions, $\eta_{\mu}^{(\nu)}(\tau)$, to the Jacobi equations (2.13) with the initial value boundary conditions

$$
\eta_{\mu}^{(\nu)}(0)=0 \quad ; \quad \dot{\eta}_{\mu}^{(\nu)}(0)=\delta_{\mu \nu} \quad, \quad(\mu, \nu=1,2,3,4)
$$

and evaluated at the endpoint $\tau=T$. Given the worldline instanton, $x^{\mathrm{cl}}(\tau)$, it is straightforward to implement this determinant computation numerically. Similarly, the free case is just given by the corresponding equations with $\Lambda_{\mu \nu}^{\text {free }} \equiv-\frac{1}{2} \delta_{\mu \nu} \frac{d^{2}}{d \tau^{2}}$, in which case $\operatorname{det}\left[\eta_{\mu \text {, free }}^{(\nu)}(T)\right]=T^{4}$, with these normalizations. In the next Section we show that for certain classes of inhomogeneous background fields it is possible to be even more explicit and find a simple analytic expression for this determinant in terms of the classical worldline instanton paths $x^{\mathrm{cl}}$ themselves. The (constant) phase factor $e^{i \theta}$ in (2.14) is determined by the Morse index [41, 45] of the operator $\Lambda$, which counts the number of times $\operatorname{det}\left[\eta_{\mu}^{(\nu)}(\tau)\right]$ vanishes in the interval from 0 to $T$. In the cases considered in this paper we find this phase factor reduces to \pm 1 , as described below. 


\section{TIME-DEPENDENT ELECTRIC FIELD}

In this section we illustrate our semiclassical procedure in a class of models where the computation can be done very explicitly. These cases 1] are those where the electric field points in a given direction in space (say the $x_{3}$ direction), and is either (i) a function only of time, or (ii) a function only of $x_{3}$. The case (i) has been widely studied in conventional WKB [33, 34], while the case (ii) has been studied using WKB instantons [36], and numerically using worldline loops [39]. We first consider case (i), that of a time-dependent electric field, where the imaginary Euclidean gauge field (corresponding to a real Minkowski electric field) can be written as

$$
A_{3}\left(x_{4}\right)=-i \frac{E}{\omega} f\left(\omega x_{4}\right)
$$

Throughout the paper we will illustrate the method explicitly using the examples of $f\left(\omega x_{4}\right)=$ $\tan \left(\omega x_{4}\right)$, corresponding to a single-pulse Minkowski electric field $E(t)=E \operatorname{sech}^{2}(\omega t)$, and $f\left(\omega x_{4}\right)=\sinh \left(\omega x_{4}\right)$, corresponding to a periodic Minkowski electric field $E(t)=E \cos (\omega t)$. Motivated by the analogy with Keldysh's classic work [35] on atomic ionization, the inhomogeneity of the background is usually characterized by the dimensionless adiabaticity parameter

$$
\gamma \equiv \frac{m \omega}{e E}
$$

\section{A. Classical solutions}

The classical equations of motion (2.6) become

$$
\begin{aligned}
& \ddot{x}_{1}=0, \\
& \ddot{x}_{2}=0, \\
& \ddot{x}_{3}=-2 e E f^{\prime}\left(\omega x_{4}\right) \dot{x}_{4}, \\
& \ddot{x}_{4}=2 e E f^{\prime}\left(\omega x_{4}\right) \dot{x}_{3} .
\end{aligned}
$$

For periodic solutions, $x_{1}^{\mathrm{cl}}$ and $x_{2}^{\mathrm{cl}}$ must be constant, so the relation (2.7) reduces to

$$
\left(\dot{x}_{3}^{\mathrm{cl}}\right)^{2}+\left(\dot{x}_{4}^{\mathrm{cl}}\right)^{2} \equiv a^{2}
$$

and we are left with an effectively two-dimensional problem in the $\left(x_{3}, x_{4}\right)$ plane. The third equation in (3.3) can be integrated immediately:

$$
\dot{x}_{3}^{\mathrm{cl}}=-\frac{2 e E}{\omega} f\left(\omega x_{4}^{\mathrm{cl}}\right) .
$$


Here we have chosen the integration constant to vanish in order to have a periodic solution. Using (3.4) we find that the remaining equation is a first-order nonlinear equation for $x_{4}^{\mathrm{cl}}$ :

$$
\dot{x}_{4}^{\mathrm{cl}}= \pm a \sqrt{1-\left(\frac{f\left(\omega x_{4}^{\mathrm{cl}}\right)}{\bar{\gamma}}\right)^{2}},
$$

where $\bar{\gamma}$ is defined as

$$
\bar{\gamma} \equiv \frac{a \omega}{2 e E}=\frac{a}{2 m} \gamma
$$

The nonlinear equation (3.6) is the same as the one considered in [1], with $\bar{\gamma}$ in place of $\gamma$, so we can use the results of [1] to write the explicit form of the solutions $\left(x_{3}^{\mathrm{cl}}, x_{4}^{\mathrm{cl}}\right)$. For example:

- For the Minkowski electric field $E(t)=E \operatorname{sech}^{2}(\omega t)$, we have $f\left(\omega x_{4}\right)=\tan \left(\omega x_{4}\right)$, and the worldline instanton loop is [1]:

$$
\begin{aligned}
& x_{3}^{\mathrm{cl}}(\tau)=\frac{1}{\omega} \frac{1}{\sqrt{1+\bar{\gamma}^{2}}} \operatorname{arcsinh}\left[\bar{\gamma} \cos \left(2 e E \sqrt{1+\bar{\gamma}^{2}}\left(\tau+\tau_{0}\right)\right)\right], \\
& x_{4}^{\mathrm{cl}}(\tau)=\frac{1}{\omega} \arcsin \left[\frac{\bar{\gamma}}{\sqrt{1+\bar{\gamma}^{2}}} \sin \left(2 e E \sqrt{1+\bar{\gamma}^{2}}\left(\tau+\tau_{0}\right)\right)\right] .
\end{aligned}
$$

Periodicity of this solution enforces the following functional relation between $T$ and $\bar{\gamma}$ (or, equivalently, between $T$ and $a$ ):

$$
T=\frac{\pi}{e E} \frac{1}{\sqrt{1+\bar{\gamma}^{2}}} .
$$

- For the Minkowski electric field $E(t)=E \cos (\omega t)$, we have $f\left(\omega x_{4}\right)=\sinh \left(\omega x_{4}\right)$, and the worldline instanton loop is [1]:

$$
\begin{aligned}
& x_{3}^{\mathrm{cl}}(\tau)=\frac{1}{\omega} \arcsin \left[\frac{\bar{\gamma}}{\sqrt{1+\bar{\gamma}^{2}}} \operatorname{cd}\left(2 e E \sqrt{1+\bar{\gamma}^{2}}\left(\tau+\tau_{0}\right) \mid \frac{\bar{\gamma}^{2}}{1+\bar{\gamma}^{2}}\right)\right], \\
& x_{4}^{\mathrm{cl}}(\tau)=\frac{1}{\omega} \operatorname{arcsinh}\left[\frac{\bar{\gamma}}{\sqrt{1+\bar{\gamma}^{2}}} \operatorname{sd}\left(2 e E \sqrt{1+\bar{\gamma}^{2}}\left(\tau+\tau_{0}\right) \mid \frac{\bar{\gamma}^{2}}{1+\bar{\gamma}^{2}}\right)\right] .
\end{aligned}
$$

Here, cd and sd are Jacobi elliptic functions [46]. Periodicity of this solution enforces the following functional relation between $T$ and $\bar{\gamma}$ (or, equivalently, between $T$ and $a$ ):

$$
T=\frac{2}{e E} \frac{\mathbf{K}\left(\frac{\bar{\gamma}^{2}}{1+\bar{\gamma}^{2}}\right)}{\sqrt{1+\bar{\gamma}^{2}}},
$$

where $\mathbf{K}(\alpha)$ denotes the elliptic quarter period [46]. 
In these classical solutions, $\tau_{0}$ is an arbitrary constant whose physical interpretation is to label the fixed point on the loop about which the fluctuations are taken, as described above. The integration over this collective coordinate is discussed below [see (3.23)].

An interesting geometric observation is that the curvature of the planar loop $\left(x_{3}^{\mathrm{cl}}(\tau), x_{4}^{\mathrm{cl}}(\tau)\right)$ at any given point on the curve is

$$
\kappa(\tau)=\frac{\dot{x}_{3} \ddot{x}_{4}-\dot{x}_{4} \ddot{x}_{3}}{\left(\dot{x}_{3}^{2}+\dot{x}_{4}^{2}\right)^{3 / 2}}=\frac{2 e}{a} E f^{\prime}\left(\omega x_{4}\right) \quad,
$$

which is proportional to the Euclidean electric field strength $E f^{\prime}\left(\omega x_{4}\right)$ evaluated at that point. Thus, for the constant electric field case the classical path is a circle, which has constant curvature, while for an inhomogeneous electric field the planar curvature changes along the loop, in such a way that it tracks the electric field. See [47] for a recent investigation of the connection between electromagnetic fields and the geometry of the associated particle trajectories.

\section{B. Fluctuation operator and its determinant}

For the time-dependent fields of the form (3.1), the fluctuation operator (2.12) can be restricted to its components in the $\left(x_{3}, x_{4}\right)$ plane:

$$
\begin{aligned}
\Lambda & =\left(\begin{array}{cc}
-\frac{1}{2} \frac{d^{2}}{d^{2} \tau} & -e E f^{\prime}\left(\omega x_{4}^{\mathrm{cl}}\right) \frac{d}{d \tau}-e E \omega f^{\prime \prime}\left(\omega x_{4}^{\mathrm{cl}}\right) \dot{x}_{4}^{\mathrm{cl}} \\
e E f^{\prime}\left(\omega x_{4}^{\mathrm{cl}}\right) \frac{d}{d \tau} & -\frac{1}{2} \frac{d^{2}}{d^{2} \tau}+e E \omega f^{\prime \prime}\left(\omega x_{4}^{\mathrm{cl}}\right) \dot{x}_{3}^{\mathrm{cl}}
\end{array}\right) \\
& =\frac{1}{2}\left(\begin{array}{cc}
-\frac{d^{2}}{d^{2} \tau} & \frac{\ddot{x}_{3}^{\mathrm{cl}}}{\ddot{x}_{4}^{\mathrm{cl}}} \frac{d}{d \tau}+\frac{d}{d \tau}\left(\frac{\ddot{x}_{3}^{\mathrm{cl}}}{\ddot{x}_{4}^{\mathrm{cl}}}\right) \\
-\frac{\ddot{x}_{3}^{\mathrm{cl}}}{\dot{x}_{4}^{\mathrm{cl}}} \frac{d}{d \tau} & -\frac{d^{2}}{d^{2} \tau}-\frac{\dot{x}_{3}^{2}}{\dot{x}_{4}^{\mathrm{ll}}} \frac{d}{d \tau}\left(\frac{\ddot{x}_{3}^{\mathrm{cl}}}{\dot{x}_{4}^{\mathrm{cl}}}\right)
\end{array}\right) .
\end{aligned}
$$

To compute the fluctuation determinant we use the semiclassical quantum mechanical path integral result (2.14). Thus, we need to find solutions to the Jacobi equations $\Lambda \eta=0$ in (2.13), satisfying the initial value conditions (2.15). Remarkably, for the fluctuation operator 
(3.13) we can write all four independent solutions to the Jacobi equations (2.13):

$$
\begin{aligned}
\phi^{(1)}(\tau) & =\left(\begin{array}{c}
1 \\
0
\end{array}\right), \\
\phi^{(2)}(\tau) & =\left(\begin{array}{c}
\dot{x}_{3}^{\mathrm{cl}}(\tau) \\
\dot{x}_{4}^{\mathrm{cl}}(\tau)
\end{array}\right), \\
\phi^{(3)}(\tau)= & \left(\begin{array}{c}
\dot{x}_{3}^{\mathrm{cl}}(\tau) \int_{0}^{\tau} d t \frac{1}{\left[\dot{x}_{4}^{\mathrm{cl}}(t)\right]^{2}}-\int_{0}^{\tau} d t \frac{\dot{x}_{3}^{\mathrm{cl}}(t)}{\left[\dot{x}_{4}^{\mathrm{cl}}(t)\right]^{2}} \\
\dot{x}_{4}^{\mathrm{cl}}(\tau) \int_{0}^{\tau} d t \frac{1}{\left[\dot{x}_{4}^{\mathrm{cl}}(t)\right]^{2}}
\end{array}\right), \\
\phi^{(4)}(\tau)= & \left(\begin{array}{c}
\dot{x}_{3}^{\mathrm{cl}}(\tau) \int_{0}^{\tau} d t \frac{\dot{x}_{3}^{\mathrm{cl}}(t)}{\left[\dot{x}_{4}^{\mathrm{cc}}(t)\right]^{2}}-a^{2} \int_{0}^{\tau} d t \frac{1}{\left[\dot{x}_{4}^{\mathrm{cl}}(t)\right]^{2}} \\
\dot{x}_{4}^{\mathrm{cl}}(\tau) \int_{0}^{\tau} d t \frac{\dot{x}_{3}^{\mathrm{cl}}(t)}{\left[\dot{x}_{4}^{\mathrm{cl}}(t)\right]^{2}}
\end{array}\right)
\end{aligned}
$$

The first zero mode $\phi^{(1)}$ corresponds to translational invariance in the $x_{3}$ direction, while the second zero mode $\phi^{(2)}$ corresponds to invariance under shifts of the starting point on the loop. The third and the fourth zero modes $\phi^{(3,4)}$ are associated with the velocity whose magnitude $a$ is a constant. The linear combinations satisfying the initial conditions (2.15) are

$$
\begin{aligned}
& \eta^{(3)}(\tau)=\dot{x}_{3}^{\mathrm{cl}}(0) \phi^{(3)}(\tau)-\phi^{(4)}(\tau) \\
& \eta^{(4)}(\tau)=\dot{x}_{4}^{\mathrm{cl}}(0) \phi^{(3)}(\tau)
\end{aligned}
$$

A simple computation shows that the fluctuation determinant is

$$
\begin{aligned}
\operatorname{Det}(\Lambda) & \equiv \operatorname{det}\left[\eta^{(3)}(T), \eta^{(4)}(T)\right] \\
& =\frac{\left[\dot{x}_{4}^{\mathrm{cl}}(0)\right]^{3}}{\dot{x}_{4}^{\mathrm{cl}}(T)}\left[a^{2} I_{1}^{2}(T)-I_{2}^{2}(T)\right],
\end{aligned}
$$

where

$$
\begin{aligned}
& I_{1}(\tau) \equiv \frac{\dot{x}_{4}^{\mathrm{cl}}(\tau)}{\dot{x}_{4}^{\mathrm{cl}}(0)} \int_{0}^{\tau} d t \frac{1}{\left[\dot{x}_{4}^{\mathrm{cl}}(t)\right]^{2}} \\
& I_{2}(\tau) \equiv \frac{\dot{x}_{4}^{\mathrm{cl}}(\tau)}{\dot{x}_{4}^{\mathrm{cl}}(0)} \int_{0}^{\tau} d t \frac{\dot{x}_{3}^{\mathrm{cl}}(t)}{\left[\dot{x}_{4}^{\mathrm{cl}}(t)\right]^{2}}
\end{aligned}
$$

For example, for the two special cases considered above, we find

- For the Minkowski electric field $E(t)=E \operatorname{sech}^{2}(\omega t)$, with $f\left(\omega x_{4}\right)=\tan \left(\omega x_{4}\right)$, the 
classical velocities (with the periodicity condition (3.9) imposed) are [1]:

$$
\begin{aligned}
& \dot{x}_{3}^{\mathrm{cl}}(\tau)=-a \frac{\sin \left(\frac{2 \pi}{T}\left(\tau+\tau_{0}\right)\right)}{\sqrt{1+\bar{\gamma}^{2} \cos ^{2}\left(\frac{2 \pi}{T}\left(\tau+\tau_{0}\right)\right)}}, \\
& \dot{x}_{4}^{\mathrm{cl}}(\tau)=a \sqrt{1+\bar{\gamma}^{2}} \frac{\cos \left(\frac{2 \pi}{T}\left(\tau+\tau_{0}\right)\right)}{\sqrt{1+\bar{\gamma}^{2} \cos ^{2}\left(\frac{2 \pi}{T}\left(\tau+\tau_{0}\right)\right)}} .
\end{aligned}
$$

Then the integrals $I_{1}(T)$ and $I_{2}(T)$ in this case can be evaluated as

$$
\begin{aligned}
& I_{1}(T)=\left(\frac{\omega}{2 e E}\right)^{2} \frac{T}{1+\bar{\gamma}^{2}(T)}, \\
& I_{2}(T)=0 .
\end{aligned}
$$

- For the Minkowski electric field $E(t)=E \cos (\omega t)$, with $f\left(\omega x_{4}\right)=\sinh \left(\omega x_{4}\right)$, the classical velocities (with the periodicity condition (3.11) imposed) are [1]:

$$
\begin{aligned}
& \dot{x}_{3}^{\mathrm{cl}}(\tau)=-a \frac{1}{\sqrt{1+\bar{\gamma}^{2}}} \operatorname{sd}\left[\frac{4}{T} \mathbf{K}\left(\frac{\bar{\gamma}^{2}}{1+\bar{\gamma}^{2}}\right)\left(\tau+\tau_{0}\right) \mid \frac{\bar{\gamma}^{2}}{1+\bar{\gamma}^{2}}\right], \\
& \dot{x}_{4}^{\mathrm{cl}}(\tau)=\operatorname{acd}\left[\frac{4}{T} \mathbf{K}\left(\frac{\bar{\gamma}^{2}}{1+\bar{\gamma}^{2}}\right)\left(\tau+\tau_{0}\right) \mid \frac{\bar{\gamma}^{2}}{1+\bar{\gamma}^{2}}\right] .
\end{aligned}
$$

Then the integrals $I_{1}(T)$ and $I_{2}(T)$ in this case can be evaluated as

$$
\begin{aligned}
& I_{1}(T)=\left(\frac{\omega}{2 e E}\right)^{2} T\left[\frac{\mathbf{K}\left(\frac{\bar{\gamma}^{2}(T)}{1+\bar{\gamma}^{2}(T)}\right)-\mathbf{E}\left(\frac{\bar{\gamma}^{2}(T)}{1+\bar{\gamma}^{2}(T)}\right)}{\bar{\gamma}^{2}(T) \mathbf{K}\left(\frac{\bar{\gamma}^{2}(T)}{1+\bar{\gamma}^{2}(T)}\right)}\right], \\
& I_{2}(T)=0 .
\end{aligned}
$$

The determinant (3.16) can be simplified using periodicity, which implies $\dot{x}_{4}^{\mathrm{cl}}(0)=\dot{x}_{4}^{\mathrm{cl}}(T)$, and the vanishing of $I_{2}(T)$. Therefore, we find the simple expression for the fluctuation determinant:

$$
\operatorname{Det}(\Lambda)=\left[\frac{2 e E}{\omega} \dot{x}_{4}^{\mathrm{cl}}(0) \bar{\gamma}(T) I_{1}(T)\right]^{2},
$$

where we have written $a=\frac{e E}{\omega} \bar{\gamma}$ in terms of $\bar{\gamma}(T)$ to stress that the periodicity condition fixes the parameter $\bar{\gamma}$ to be a particular function of $T$, as in (3.9) and (3.11), for example.

Now recall that we still need to evaluate the 4-dimensional space-time integral over the fixed point on the closed loops:

$$
\int d^{4} x^{(0)} \equiv \int d x_{1}(0) d x_{2}(0) d x_{3}(0) d x_{4}(0)=V_{3} \int d \tau_{0} \dot{x}_{4}^{\mathrm{cl}}(0)
$$


where $V_{3}$ is the 3 -space volume. Observe that this factor of $\dot{x}_{4}^{\text {cl }}(0)$ cancels against the same factor in $\sqrt{\operatorname{Det}(\Lambda)}$, so that the spacetime integration effectively contributes a 3-volume factor $V_{3}$, and a factor of $\frac{T}{2}$. This last factor is just the collective coordinate contribution arising from invariance under shifts of the starting point on the loop, which gives rise to the second of the zero modes in (3.14).

Finally, to fix the phase factor in (2.14) we need the Morse index of the fluctuation operator $\Lambda$. This can be evaluate either as the number of negative eigenvalues of the operator $\Lambda$, or as the number of times $\operatorname{det}\left[\eta_{\mu}^{(\nu)}(\tau)\right]$ vanishes in the interval from 0 to $T$. We find that for the time dependent fields of the form discussed here, the Morse index is 2, leading to a phase factor $e^{-i 2 \pi / 2}=-1$. Thus, collecting all the pieces, we see that the semiclassical approximation (2.14) to the quantum mechanical path integral leads to :

$$
\Gamma_{\mathrm{Eucl}}^{\mathrm{semi}} \approx V_{3} \frac{1}{(4 \pi)^{2}} \frac{\omega}{4 e E} \int_{0}^{\infty} \frac{d T}{T} \frac{\exp \left[-\left(S\left[x^{\mathrm{cl}}\right](T)+m^{2} T\right)\right]}{\bar{\gamma}(T) I_{1}(T)}
$$

\section{Extracting the Nonperturbative Imaginary Part}

So far we have been computing the Euclidean effective action, using the worldline expression (2.1). We relate this to the imaginary part of the physical Minkowski effective action according to the following conventions:

$$
e^{i S_{\mathrm{Mink}}}=e^{i \int d t L_{\mathrm{Mink}}}=e^{\int d y_{4} L_{\mathrm{Mink}}}=e^{-S_{\mathrm{Eucl}}}=e^{-\int d y_{4} L_{\mathrm{Eucl}}}
$$

Thus we identify $y_{4}=i t, L_{\mathrm{Mink}}=-L_{\mathrm{Eucl}}$, and

$$
\Gamma_{\text {Mink }}=i \Gamma_{\text {Eucl }}
$$

Some care is needed for extracting the desired Minkowski imaginary part:

$$
\begin{aligned}
\operatorname{Im} \Gamma_{\text {Mink }} & =\operatorname{Im} \int d^{3} y \int d t \mathcal{L}_{\text {Mink }}(t, \vec{y}) \\
& =-\operatorname{Im} \int d^{3} y \int d t \mathcal{L}_{\text {Eucl }}(i t, \vec{y}) \\
& =\operatorname{Re} \int d^{3} y i \int d t \mathcal{L}_{\text {Eucl }}(i t, \vec{y}) \\
& =\operatorname{Re} \int d^{3} y \int_{\mathcal{C}} d y_{4} \mathcal{L}_{\text {Eucl }}\left(y_{4}, \vec{y}\right) \\
& =\operatorname{Re} \Gamma_{\text {Eucl }, \mathcal{C}},
\end{aligned}
$$


where the contour $\mathcal{C}$ goes along the imaginary axis from $-i \infty$ to $i \infty$. For instance, for time-independent electric background fields, we obtain

$$
\operatorname{Im} \Gamma_{\text {Mink }} \equiv \int d t \operatorname{Im} L_{\text {Mink }}=\operatorname{Re} \int_{\mathcal{C}} d y_{4} L_{\mathrm{Eucl}}=-\int d t \operatorname{Im} L_{\mathrm{Eucl}},
$$

such that the imaginary part of the Minkowski Lagrange function is given by (minus) the imaginary part of the Euclidean Lagrange function (note that $L=\int d^{3} y \mathcal{L}$ ).

On the other hand, for time-dependent electric fields for which the $y_{4}$ contour $\mathcal{C}$ can be rotated onto the real axis, we find

$$
\operatorname{Im} \Gamma_{\text {Mink }}=\operatorname{Re} \int_{\mathcal{C}} d y_{4} L_{\text {Eucl }}\left(y_{4}\right)=\operatorname{Re} \int_{-\infty}^{\infty} d y_{4} L_{\text {Eucl }}\left(y_{4}\right)=\operatorname{Re} \Gamma_{\text {Eucl }} .
$$

For the electric fields considered in the present work, the contour can indeed be rotated, since worldline instantons extending to $y_{4} \rightarrow \pm \infty$ yield a vanishing contribution that drops off sufficiently fast at complex infinity. Thus to find the nonperturbative imaginary part of the Minkowski effective action in the semiclassical approximation, we need to extract the real part of the propertime integral expression in (3.24).

\section{The $T$ integral}

In general, the $T$ integral in the semiclassical expression (3.24) cannot be done analytically. However, in the weak field limit the physical nonperturbative part may be extracted directly using a steepest descents approximation, by evaluating the $T$ integral in the vicinity of a critical point. To do so, we study the exponent in (3.24) :

$$
\Delta(T)=S\left[x^{\mathrm{cl}}\right](T)+m^{2} T
$$

This notation emphasizes the fact that the action $S\left[x^{\mathrm{cl}}\right]$, evaluated on the worldline instanton path $x^{\mathrm{cl}}(\tau)$, is a function of $T$. Before considering the general case, we illustrate with the example of the Minkowski electric field $E(t)=E \operatorname{sech}^{2}(\omega t)$. Then, using the worldline instanton $x_{\mu}^{\mathrm{cl}}(\tau)$ found in (3.8), we find

$$
\begin{aligned}
\Delta(T) & =\frac{\pi^{2}}{\omega^{2} T}\left(1-\frac{e E T}{\pi}\right)^{2}+m^{2} T \\
& =\frac{m^{2} \pi}{e E}\left[\frac{e E T}{\pi}\left(\frac{1+\gamma^{2}}{\gamma^{2}}\right)+\frac{1}{\gamma^{2}} \frac{\pi}{e E T}-\frac{2}{\gamma^{2}}\right],
\end{aligned}
$$


where $\gamma$ is defined in (3.2). And we have used the relation (3.9) between $T$ and $\bar{\gamma}$, which follows from the periodicity of the solution. This can be written as

$$
\bar{\gamma}(T)=\frac{\pi}{e E T} \sqrt{1-\left(\frac{e E T}{\pi}\right)^{2}} .
$$

Using this, we can express $I_{1}(T)$ in (3.19) directly as a function of $T$ :

$$
I_{1}(T)=\left(\frac{\omega}{2 \pi}\right)^{2} T^{3}
$$

Thus,

$\Gamma_{\mathrm{Eucl}}^{\mathrm{semi}} \approx V_{3} \frac{1}{16 \pi \omega} \int_{0}^{\infty} \frac{d T}{T^{3} \sqrt{1-\left(\frac{e E T}{\pi}\right)^{2}}} \exp \left\{-\frac{m^{2} \pi}{e E}\left[\frac{e E T}{\pi}\left(\frac{1+\gamma^{2}}{\gamma^{2}}\right)+\frac{1}{\gamma^{2}} \frac{\pi}{e E T}-\frac{2}{\gamma^{2}}\right]\right\}$.

Observe that there is a branch cut along part of the real $T$ axis, so the integral has both a real and an imaginary part. As explained in the previous section, to obtain the physical nonperturbative imaginary part of the Minkowski effective action we need the real part of (3.34). The branch point occurs at

$$
T_{b}=\frac{\pi}{e E}
$$

The imaginary part of (3.34) comes from an integral across the cut, which extends from $T_{b}$ to infinity, but we are instead interested in the contribution from the region to the left of the branch point. Observe that it is natural to rescale $T$ as $\frac{e E T}{\pi}$, so that in the weak field limit $E \rightarrow 0$, we expect a dominant contribution from the vicinity of critical points of $\Delta(T)$. From (3.31) we find the critical point of the exponent is at

$$
T_{c}=\frac{\pi}{e E} \frac{1}{\sqrt{1+\gamma^{2}}}
$$

which falls to the left of the branch point, for all frequencies $\omega$ (i.e., all $\gamma$ ). So this critical point produces the required dominant contribution. Comparing (3.9) and (3.36), we see that the critical point occurs when

$$
\bar{\gamma}\left(T_{c}\right)=\gamma
$$

Recalling the definition (3.7) of $\bar{\gamma}$, we see that the critical point occurs when $a=2 m$, which is precisely the value used in [1] when the propertime $T$ integral was done first. In fact, this property is general, as we show in the next section. 
At the critical point, the exponent is

$$
\Delta\left(T_{c}\right)=\frac{m^{2} \pi}{e E}\left(\frac{2}{1+\sqrt{1+\gamma^{2}}}\right)
$$

The critical point is a minimum since

$$
\Delta^{\prime \prime}\left(T_{c}\right)=\frac{2 e E m^{2}}{\pi} \frac{\left(1+\gamma^{2}\right)^{3 / 2}}{\gamma^{2}},
$$

which is positive. Thus, we can use Laplace's method to approximate the integral in the weak field limit, leading to:

$$
\begin{aligned}
{\left[\operatorname{Im} \Gamma_{\text {Mink }}^{\mathrm{semi}}\right]_{E(t)=E \operatorname{sech}^{2}(\omega t)} } & \approx V_{3}^{\mathrm{Mink}} \frac{1}{(4 \pi)^{2}} \frac{\omega}{4 e E} \sqrt{\frac{2 \pi}{\Delta^{\prime \prime}\left(T_{c}\right)}} \frac{e^{-\Delta\left(T_{c}\right)}}{T_{c} \bar{\gamma}\left(T_{c}\right) I_{1}\left(T_{c}\right)} \\
& =V_{3}^{\mathrm{Mink}} \frac{(e E)^{5 / 2}}{16 \pi^{3} m \omega}\left(1+\gamma^{2}\right)^{5 / 4} \exp \left[-\frac{m^{2} \pi}{e E}\left(\frac{2}{1+\sqrt{1+\gamma^{2}}}\right)\right]
\end{aligned}
$$

This should be compared to the locally constant field (LCF) approximation in (3.27):

$$
\begin{aligned}
{\left[\operatorname{Im} \Gamma_{\text {Mink }}^{\mathrm{LCF}}\right]_{E(t)=E \operatorname{sech}^{2}(\omega t)} } & \approx V_{3}^{\mathrm{Mink}} \frac{e^{2} E^{2}}{16 \pi^{3}} \int_{-\infty}^{\infty} d t \operatorname{sech}^{4}(\omega t) \exp \left[-\frac{m^{2} \pi}{e E} \cosh ^{2}(\omega t)\right] \\
& \sim V_{3}^{\mathrm{Mink}} \frac{(e E)^{5 / 2}}{16 \pi^{3} m \omega} \exp \left[-\frac{m^{2} \pi}{e E}\right] .
\end{aligned}
$$

Here, in the second line of (3.41) we have made the same weak field limit approximation as was made in (3.40). Then (3.41) agrees perfectly with the static limit $(\gamma \rightarrow 0)$ of our semiclassical result (3.40). The semiclassical result (3.40) also agrees with Popov's WKB result [34] for this time dependent electric field, and with the Borel analysis of the resummed derivative expansion [48]. Note that the temporal inhomogeneity of the field enhances the local pair production rate, as discussed in [1, 34].

\section{E. General case}

We now show that the example given in the previous section is quite general for time

dependent electric fields of the form (3.1). Using the classical equations of motion (3.3), we can write

$$
\begin{aligned}
S\left[x^{\mathrm{cl}}\right](T) & =\int_{0}^{T} d \tau\left\{\frac{1}{4}\left[\left(\dot{x}_{3}^{\mathrm{cl}}\right)^{2}+\left(\dot{x}_{4}^{\mathrm{cl}}\right)^{2}\right]+\frac{e E}{\omega} f\left(\omega x_{4}^{\mathrm{cl}}\right) \dot{x}_{3}^{\mathrm{cl}}\right\} \\
& =-\frac{a^{2}}{4} T+\frac{1}{2} \int_{0}^{T} d \tau\left(\dot{x}_{4}^{\mathrm{cl}}\right)^{2} .
\end{aligned}
$$


Thus, the exponent $\Delta(T)$ can be expressed as a (complicated) function of $T$ :

$$
\Delta(T)=m^{2} T\left(1-\frac{\bar{\gamma}^{2}(T)}{\gamma^{2}}\right)+\frac{\pi m^{2}}{e E} \frac{\bar{\gamma}^{2}(T)}{\gamma^{2}} g\left(\bar{\gamma}^{2}\right),
$$

where we have defined the important function $g$ :

$$
g\left(\bar{\gamma}^{2}\right) \equiv \frac{2}{\pi} \int_{-1}^{1} \frac{d y \sqrt{1-y^{2}}}{\left|f^{\prime}\right|}
$$

In this definition of the function $g$, we have written $y=\frac{f\left(\omega x_{4}^{\mathrm{cl}}\right)}{\bar{\gamma}}$, and $f^{\prime}$ means the derivative is re-expressed as a function of $y$. With the same notation, the periodicity condition can be expressed in general as

$$
T=\frac{1}{e E} \int_{-1}^{1} \frac{d y}{\left|f^{\prime}\right| \sqrt{1-y^{2}}} \equiv \frac{\pi}{e E} P\left(\bar{\gamma}^{2}\right),
$$

which determines $\bar{\gamma}$ as a function of $T$. Note that the two functions, $g$ and $P$ are related as follows:

$$
P(z)=\frac{d}{d z}(z g(z))
$$

For example:

- For the Minkowski electric field $E(t)=E \operatorname{sech}^{2}(\omega t)$, we have $f\left(\omega x_{4}\right)=\tan \left(\omega x_{4}\right)$, so that

$$
f^{\prime}\left(\omega x_{4}\right)=\sec ^{2}\left(\omega x_{4}\right)=1+\bar{\gamma}^{2} y^{2}
$$

Then the exponent (3.43) involves the function

$$
g\left(\bar{\gamma}^{2}\right)=\frac{2}{\pi} \int_{-1}^{1} \frac{d y \sqrt{1-y^{2}}}{\left(1+\bar{\gamma}^{2} y^{2}\right)}=\frac{2}{1+\sqrt{1+\bar{\gamma}^{2}}},
$$

and the periodicity condition (3.45) involves the function

$$
P\left(\bar{\gamma}^{2}\right)=\frac{1}{\pi} \int_{-1}^{1} \frac{d y}{\left(1+\bar{\gamma}^{2} y^{2}\right) \sqrt{1-y^{2}}}=\frac{1}{\sqrt{1+\bar{\gamma}^{2}}} .
$$

- For the Minkowski electric field $E(t)=E \cos (\omega t)$, we have $f\left(\omega x_{4}\right)=\sinh \left(\omega x_{4}\right)$, so that

$$
f^{\prime}\left(\omega x_{4}\right)=\cosh \left(\omega x_{4}\right)=\sqrt{1+\bar{\gamma}^{2} y^{2}} .
$$

Then the exponent (3.43) involves the function

$$
g\left(\bar{\gamma}^{2}\right)=\frac{2}{\pi} \int_{-1}^{1} \frac{d y \sqrt{1-y^{2}}}{\sqrt{1+\bar{\gamma}^{2} y^{2}}}=\frac{4}{\pi} \frac{\sqrt{1+\bar{\gamma}^{2}}}{\bar{\gamma}^{2}}\left[\mathbf{K}\left(\frac{\bar{\gamma}^{2}}{1+\bar{\gamma}^{2}}\right)-\mathbf{E}\left(\frac{\bar{\gamma}^{2}}{1+\bar{\gamma}^{2}}\right)\right]
$$


and the periodicity condition (3.45) involves the function

$$
P\left(\bar{\gamma}^{2}\right)=\frac{1}{\pi} \int_{-1}^{1} \frac{d y}{\sqrt{1+\bar{\gamma}^{2} y^{2}} \sqrt{1-y^{2}}}=\frac{2}{\pi} \frac{1}{\sqrt{1+\bar{\gamma}^{2}}} \mathbf{K}\left(\frac{\bar{\gamma}^{2}}{1+\bar{\gamma}^{2}}\right) .
$$

We identify the critical point of the exponent as follows. From (3.43) - (3.45) it follows that the derivative with respect to $T$ takes a remarkably simple form:

$$
\frac{d \Delta(T)}{d T}=m^{2}\left[1-\frac{\bar{\gamma}^{2}(T)}{\gamma^{2}}\right] .
$$

Thus, the critical point $T_{c}$ of the exponent occurs at $T$ such that $\bar{\gamma}\left(T_{c}\right)=\gamma$ [as was found before in (3.37)], which determines $T_{c}$ as a particular function of $\gamma$ :

$$
T_{c}=\frac{\pi}{e E} P\left(\gamma^{2}\right)
$$

Evaluating the exponent at this critical point yields

$$
\Delta\left(T_{c}\right)=\frac{m^{2} \pi}{e E} g\left(\gamma^{2}\right)
$$

which is the exponent derived in [1]. From (3.53) and (3.45), the second derivative of the exponent, evaluated at the critical point, is

$$
\left[\frac{d^{2} \Delta(T)}{d T^{2}}\right]_{T=T_{c}}=-\frac{m^{2} e E}{\pi} \frac{1}{\gamma^{2} \frac{d P\left(\gamma^{2}\right)}{d\left(\gamma^{2}\right)}} .
$$

The final ingredient for the semiclassical evaluation of the pair production rate is to evaluate the determinant prefactor at $T_{c}$, for which we find the following simple expression, involving the same function $P\left(\gamma^{2}\right)$ :

$$
I_{1}\left(T_{c}\right)=\left[\int_{0}^{T} \frac{d \tau}{\left(\dot{x}_{4}^{\mathrm{cl}}\right)^{2}}\right]_{T=T_{c}}=-\frac{\pi}{2 m^{2} e E} \gamma^{2} \frac{d P\left(\gamma^{2}\right)}{d\left(\gamma^{2}\right)}
$$

Thus, our final semiclassical approximation for the nonperturbative imaginary part of the Minkowski effective action for a time-dependent electric field background is:

$$
\begin{aligned}
\operatorname{Im} \Gamma_{\text {Mink }}^{\text {semi }} & \approx V_{3}^{\text {Mink }} \frac{1}{(4 \pi)^{2}} \frac{\omega}{4 e E} \sqrt{\frac{2 \pi}{\Delta^{\prime \prime}\left(T_{c}\right)}} \frac{e^{-\Delta\left(T_{c}\right)}}{T_{c} \bar{\gamma}\left(T_{c}\right) I_{1}\left(T_{c}\right)} \\
& =V_{3}^{\text {Mink }} \frac{\sqrt{2}(e E)^{5 / 2}}{32 \pi^{3} m \omega} \frac{1}{\frac{d}{d\left(\gamma^{2}\right)}\left(\gamma^{2} g\left(\gamma^{2}\right)\right) \sqrt{-\frac{d^{2}}{d\left(\gamma^{2}\right)^{2}}\left(\gamma^{2} g\left(\gamma^{2}\right)\right)}} \exp \left[-\frac{m^{2} \pi}{e E} g\left(\gamma^{2}\right)\right],
\end{aligned}
$$

where $g\left(\gamma^{2}\right)$ is the function defined in (3.44), evaluated at $\bar{\gamma}=\gamma$. Recalling (3.46) that $P\left(\gamma^{2}\right)$ is completely determined by the function $g\left(\gamma^{2}\right)$, we note the important fact that the semiclassical approximation (3.58) is expressed entirely in terms of the single function $g\left(\gamma^{2}\right)$.

For example: 
- For $E(t)=E \operatorname{sech}^{2}(\omega t)$, we find the result in (3.40).

- For $E(t)=E \cos (\omega t)$, we find

$\operatorname{Im} \Gamma_{\text {Mink }}^{\mathrm{semi}} \approx V_{3}^{\mathrm{Mink}} \frac{\sqrt{2 \pi}(e E)^{3 / 2}}{64 \pi^{2}} \frac{\left(1+\gamma^{2}\right)^{3 / 4} \exp \left\{-\frac{4 m^{2}}{e E} \frac{\sqrt{1+\gamma^{2}}}{\gamma^{2}}\left[\mathbf{K}\left(\frac{\gamma^{2}}{1+\gamma^{2}}\right)-\mathbf{E}\left(\frac{\gamma^{2}}{1+\gamma^{2}}\right)\right]\right\}}{\mathbf{K}\left(\frac{\gamma^{2}}{1+\gamma^{2}}\right) \sqrt{\mathbf{K}\left(\frac{\gamma^{2}}{1+\gamma^{2}}\right)-\mathbf{E}\left(\frac{\gamma^{2}}{1+\gamma^{2}}\right)}}$.

Once again, in the static limit $(\gamma \rightarrow 0)$, this reduces to the locally constant field approximation result

$$
\begin{aligned}
\operatorname{Im} \Gamma_{\text {Mink }}^{\mathrm{LCF}} & \approx V_{3}^{\mathrm{Mink}} \frac{e^{2}}{16 \pi^{3}} \int_{-\frac{\pi}{2 \omega}}^{\frac{\pi}{2 \omega}} d t E^{2} \cos ^{2}(\omega t) \exp \left[-\frac{m^{2} \pi}{e E|\cos (\omega t)|}\right] \\
& \sim V_{3}^{\mathrm{Mink}} \frac{\sqrt{2}(e E)^{5 / 2}}{16 \pi^{3} m \omega} \exp \left[-\frac{m^{2} \pi}{e E}\right] .
\end{aligned}
$$

The result (3.59) also agrees with Popov's WKB analysis, after some (presumably typographical) errors are corrected in [34].

We stress the simplicity and versatility of the result (3.58). It means that for background fields where the gauge field (and hence the electric field) is a function of just one space-time coordinate, one does not even have to find the explicit form of the semiclassical worldline instanton path which dominates the functional integral. Instead, one simply needs to compute the function $g\left(\gamma^{2}\right)$ [defined in (3.44) $]$, and its first few derivatives. Even if this cannot be done in closed-form, it could be done numerically. As a final example we can take the time-dependent Minkowski electric field

$$
E(t)=\frac{E}{\left(1+(\omega t)^{2}\right)^{3 / 2}}
$$

for which the Euclidean gauge function is

$$
f\left(\omega x_{4}\right)=\frac{\omega x_{4}}{\sqrt{1-\left(\omega x_{4}\right)^{2}}} .
$$

Then the function $g\left(\gamma^{2}\right)$ in (3.44) is

$$
g\left(\gamma^{2}\right)=\frac{2}{\pi} \int_{-1}^{1} d y \frac{\sqrt{1-y^{2}}}{\left(1+\gamma^{2} y^{2}\right)^{3 / 2}}=\frac{4}{\pi \gamma^{2}}\left[\mathbf{E}\left(-\gamma^{2}\right)-\mathbf{K}\left(-\gamma^{2}\right)\right]
$$


Then the semiclassical imaginary part of the Minkowski effective action is

$$
\operatorname{Im} \Gamma_{\text {Mink }}^{\text {semi }} \approx V_{3}^{\operatorname{Mink}} \frac{\sqrt{2 \pi}(e E)^{3 / 2}}{64 \pi^{2}} \frac{\left(1+\gamma^{2}\right)^{3 / 4} \exp \left\{-\frac{4 m^{2}}{e E \gamma^{2}}\left[\mathbf{E}\left(-\gamma^{2}\right)-\mathbf{K}\left(-\gamma^{2}\right)\right]\right\}}{\mathbf{E}\left(-\gamma^{2}\right) \sqrt{\left(1+\gamma^{2}\right) \mathbf{K}\left(-\gamma^{2}\right)-\left(1-\gamma^{2}\right) \mathbf{E}\left(-\gamma^{2}\right)}}
$$

Note that while we could have computed the explicit worldline instanton path for this background, it was in fact not necessary in order to compute the semiclassical imaginary part of the Minkowski effective action. In the static limit $(\gamma \rightarrow 0)$, this reduces to the locally constant field approximation result

$$
\begin{aligned}
\operatorname{Im} \Gamma_{\text {Mink }}^{\mathrm{LCF}} & \approx V_{3}^{\mathrm{Mink}} \frac{e^{2}}{16 \pi^{3}} \int_{-\infty}^{\infty} d t \frac{E^{2}}{\left[1+(\omega t)^{2}\right]^{3}} \exp \left\{-\frac{m^{2} \pi}{e E}\left[1+(\omega t)^{2}\right]^{3 / 2}\right\} \\
& \sim V_{3}^{\mathrm{Mink}} \frac{\sqrt{2}(e E)^{5 / 2}}{16 \pi^{3} \sqrt{3} m \omega} \exp \left[-\frac{m^{2} \pi}{e E}\right] .
\end{aligned}
$$

The result (3.64) also agrees with Popov's WKB analysis, after some (presumably typographical) errors are corrected in [34].

\section{SPATIALLY INHOMOGENEOUS ELECTRIC FIELDS}

As discussed already in [1], spatially inhomogeneous electric fields that are functions of a single spatial coordinate, say $x_{3}$, can be treated analogously. Consider the class of spatially inhomogeneous electric fields with Euclidean gauge field

$$
A_{4}\left(x_{3}\right)=-i \frac{E}{k} f\left(k x_{3}\right) \text {. }
$$

For example, the single-bump Minkowski electric field $E\left(x_{3}\right)=E \operatorname{sech}^{2}\left(k x_{3}\right)$ has $f\left(k x_{3}\right)=$ $\tanh \left(k x_{3}\right)$, while the periodic Minkowski electric field $E\left(x_{3}\right)=E \cos \left(k x_{3}\right)$ has $f\left(k x_{3}\right)=$ $\sin \left(k x_{3}\right)$. Define the spatial adiabaticity parameter

$$
\tilde{\gamma}=\frac{m k}{e E} .
$$

The entire analysis of worldline instantons can be repeated as in the time-dependent case of Section III] although the $T$ integral requires an analytic continuation to the complex plane in order to be evaluated by steepest descents. In fact, the final results for the imaginary part of the Minkowski effective action can be obtained from those of the corresponding time-dependent system by the analytic continuation:

$$
\gamma \rightarrow i \tilde{\gamma}
$$


Thus, our semiclassical approximation for the nonperturbative imaginary part of the Minkowski effective action for a space-dependent electric field background with gauge function (4.1) is:

$$
\operatorname{Im} \Gamma_{\text {Mink }}^{\text {semi }} \approx\left(V_{2} \mathcal{T}\right)^{\operatorname{Mink}} \frac{\sqrt{2}(e E)^{5 / 2}}{32 \pi^{3} m k} \frac{\exp \left[-\frac{m^{2} \pi}{e E} \tilde{g}\left(\tilde{\gamma}^{2}\right)\right]}{\frac{d}{d\left(\tilde{\gamma}^{2}\right)}\left(\tilde{\gamma}^{2} \tilde{g}\left(\tilde{\gamma}^{2}\right)\right) \sqrt{\frac{d^{2}}{d\left(\tilde{\gamma}^{2}\right)^{2}}\left(\tilde{\gamma}^{2} \tilde{g}\left(\tilde{\gamma}^{2}\right)\right)}},
$$

where $\tilde{g}\left(\tilde{\gamma}^{2}\right)$ is the function defined by

$$
\tilde{g}\left(\tilde{\gamma}^{2}\right)=\frac{2}{\pi} \int_{-1}^{1} d y \frac{\sqrt{1-y^{2}}}{\left|f^{\prime}\right|}
$$

where we have written $y=\frac{f\left(k x_{4}^{\mathrm{cl}}\right)}{\tilde{\gamma}}$, and $f^{\prime}$ means the derivative is re-expressed as a function of $y$. For example:

- For the Minkowski electric field $E\left(x_{3}\right)=E \operatorname{sech}^{2}\left(k x_{3}\right)$, we have $f\left(k x_{3}\right)=\tanh \left(k x_{3}\right)$, and $f^{\prime}\left(k x_{3}\right)=\operatorname{sech}^{2}\left(k x_{3}\right)=1-\tilde{\gamma}^{2} y^{2}$. Thus,

$$
\tilde{g}\left(\tilde{\gamma}^{2}\right)=\frac{2}{\pi} \int_{-1}^{1} \frac{d y \sqrt{1-y^{2}}}{\left(1-\tilde{\gamma}^{2} y^{2}\right)}=\frac{2}{1+\sqrt{1-\tilde{\gamma}^{2}}} .
$$

The imaginary part of the Minkowski effective action is

$$
\operatorname{Im} \Gamma_{\text {Mink }}^{\text {semi }}=\left(V_{2} \mathcal{T}\right)^{\operatorname{Mink}} \frac{(e E)^{5 / 2}}{16 \pi^{3} m k}\left(1-\tilde{\gamma}^{2}\right)^{5 / 4} \exp \left[-\frac{m^{2} \pi}{e E}\left(\frac{2}{1+\sqrt{1-\tilde{\gamma}^{2}}}\right)\right]
$$

The corresponding locally constant field approximation is

$$
\begin{aligned}
\operatorname{Im} \Gamma_{\text {Mink }}^{\mathrm{LCF}} & =\left(V_{2} \mathcal{T}\right)^{\operatorname{Mink}} \frac{e^{2} E^{2}}{16 \pi^{3}} \int_{-\infty}^{\infty} d x_{3} \operatorname{sech}^{4}\left(k x_{3}\right) \exp \left[-\frac{m^{2} \pi}{e E} \cosh ^{2}\left(k x_{3}\right)\right] \\
& \sim\left(V_{2} \mathcal{T}\right)^{\operatorname{Mink}} \frac{(e E)^{5 / 2}}{16 \pi^{3} m k} \exp \left[-\frac{m^{2} \pi}{e E}\right] .
\end{aligned}
$$

The ratio of the semiclassical answer (4.7) to the LCF approximation (4.8) is plotted in Figure 1, along with the exact result of [49] and the numerical results of [39]. The agreement between the semiclassical expression and the numerical results is excellent. This is especially true given that the numerical data is from a system with $\frac{e E}{m^{2}}=$ 1, which is far from the weak field limit in which the semiclassical expression was derived. We also comment that (4.4) and (4.7) agree with the quantum mechanical instanton result of Kim and Page [36, 50], after a gaussian integration over energy and momentum. 


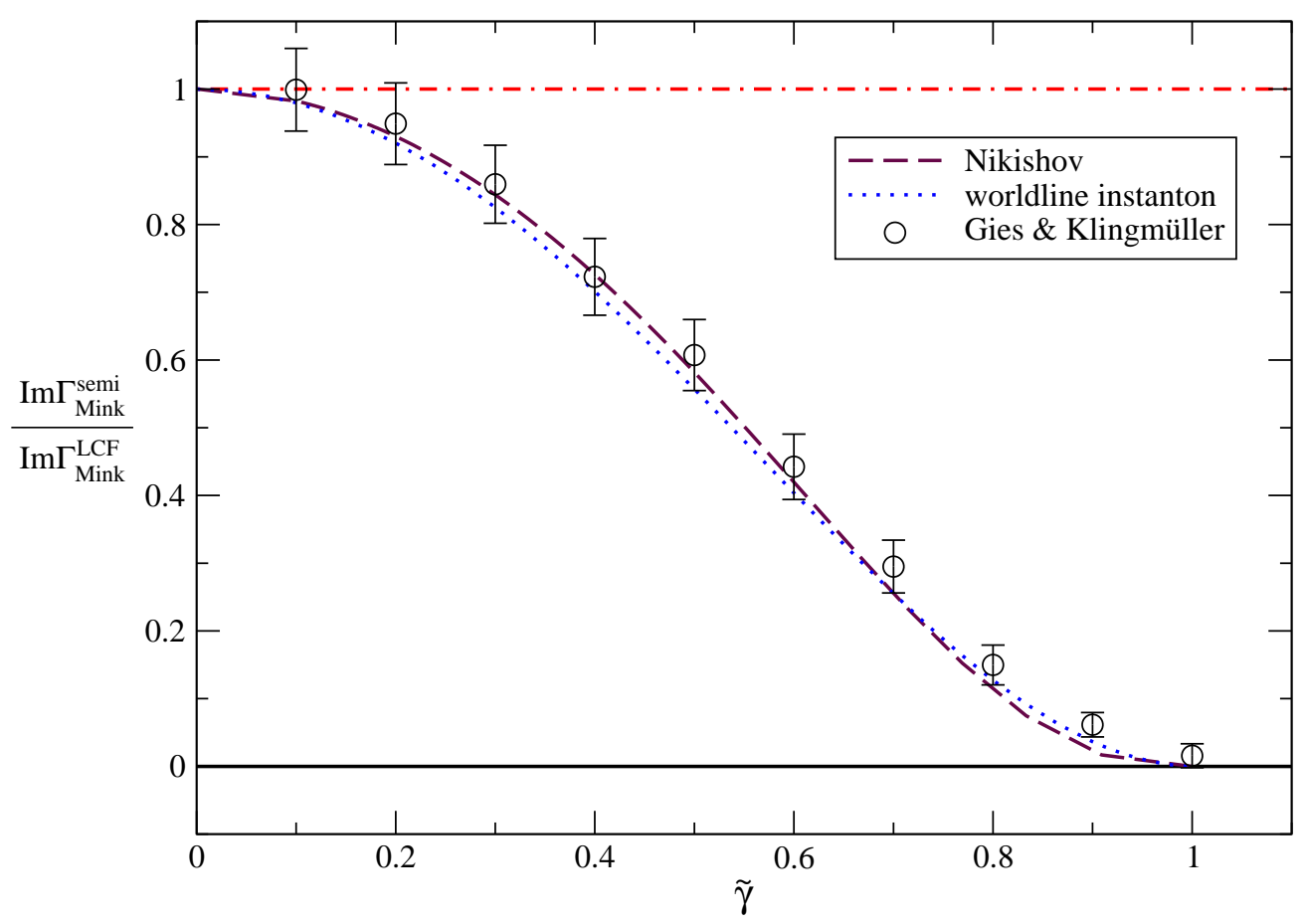

FIG. 1: The dotted line plots the ratio of our semiclassical worldline instanton expression (4.7) to the weak field limit of the corresponding locally constant field approximation (4.8). The dashed line is the same ratio using a numerical integration of the exact expression, derived from Nikishov's exact result in [49] (see also 36]). The circles represent the numerical worldline results of Gies and Klingmüller [39], which were evaluated for $\frac{e E}{m^{2}}=1$. Note that the agreement is excellent, even though it is far from the weak field limit.

- For the Minkowski electric field $E\left(x_{3}\right)=E \cos \left(k x_{3}\right)$, we have $f\left(k x_{3}\right)=\sin \left(k x_{3}\right)$, and

$$
\begin{aligned}
& f^{\prime}\left(k x_{3}\right)=\cos \left(k x_{3}\right)=\sqrt{1-\tilde{\gamma}^{2} y^{2}} \text {. Thus } \\
& \quad \tilde{g}\left(\tilde{\gamma}^{2}\right)=\frac{2}{\pi} \int_{-1}^{1} \frac{d y \sqrt{1-y^{2}}}{\sqrt{1-\tilde{\gamma}^{2} y^{2}}}=\frac{4 \sqrt{1-\tilde{\gamma}^{2}}}{\pi \tilde{\gamma}^{2}}\left[\mathbf{E}\left(\frac{-\tilde{\gamma}^{2}}{1-\tilde{\gamma}^{2}}\right)-\mathbf{K}\left(\frac{-\tilde{\gamma}^{2}}{1-\tilde{\gamma}^{2}}\right)\right] .
\end{aligned}
$$

The imaginary part of the Minkowski effective action is

$$
\operatorname{Im} \Gamma_{\text {Mink }}^{\mathrm{semi}} \approx\left(V_{2} \mathcal{T}\right)^{\operatorname{Mink}} \frac{\sqrt{2 \pi}(e E)^{3 / 2}}{64 \pi^{2}} \frac{\left(1-\tilde{\gamma}^{2}\right)^{3 / 4} \exp \left\{-\frac{4 m^{2}}{e E} \frac{\sqrt{1-\tilde{\gamma}^{2}}}{\tilde{\gamma}^{2}}\left[\mathbf{E}\left(\frac{-\tilde{\gamma}^{2}}{1-\tilde{\gamma}^{2}}\right)-\mathbf{K}\left(\frac{-\tilde{\gamma}^{2}}{1-\tilde{\gamma}^{2}}\right)\right]\right\}}{\mathbf{K}\left(\frac{-\tilde{\gamma}^{2}}{1-\tilde{\gamma}^{2}}\right) \sqrt{\mathbf{E}\left(\frac{-\tilde{\gamma}^{2}}{1-\tilde{\gamma}^{2}}\right)-\mathbf{K}\left(\frac{-\tilde{\gamma}^{2}}{1-\tilde{\gamma}^{2}}\right)}} .
$$

- For the Minkowski electric field $E\left(x_{3}\right)=\frac{E}{\left[1+\left(k x_{3}\right)^{2}\right]^{3 / 2}}$, we have $f\left(k x_{3}\right)=\frac{k x_{3}}{\sqrt{1+\left(k x_{3}\right)^{2}}}$, and 


$$
\begin{aligned}
& f^{\prime}\left(k x_{3}\right)=\left(1-\tilde{\gamma}^{2} y^{2}\right)^{3 / 2} \text {. Thus, } \\
& \qquad \tilde{g}\left(\tilde{\gamma}^{2}\right)=\frac{2}{\pi} \int_{-1}^{1} d y \frac{\sqrt{1-y^{2}}}{\left(1-\tilde{\gamma}^{2} y^{2}\right)^{3 / 2}}=\frac{4}{\pi \tilde{\gamma}^{2}}\left[\mathbf{K}\left(\tilde{\gamma}^{2}\right)-\mathbf{E}\left(\tilde{\gamma}^{2}\right)\right] .
\end{aligned}
$$

Then the semiclassical imaginary part of the Minkowski effective action is

$$
\operatorname{Im} \Gamma_{\text {Mink }}^{\text {semi }} \approx\left(V_{2} \mathcal{T}\right)^{\operatorname{Mink}} \frac{\sqrt{2 \pi}(e E)^{3 / 2}}{64 \pi^{2}} \frac{\left(1-\tilde{\gamma}^{2}\right)^{3 / 4} \exp \left\{-\frac{4 m^{2}}{e E \tilde{\gamma}^{2}}\left[\mathbf{K}\left(\tilde{\gamma}^{2}\right)-\mathbf{E}\left(\tilde{\gamma}^{2}\right)\right]\right\}}{\mathbf{E}\left(\tilde{\gamma}^{2}\right) \sqrt{\left(1+\tilde{\gamma}^{2}\right) \mathbf{E}\left(\tilde{\gamma}^{2}\right)-\left(1-\tilde{\gamma}^{2}\right) \mathbf{K}\left(\tilde{\gamma}^{2}\right)}}
$$

Note that while we could have computed the explicit worldline instanton path for this background, it was in fact not necessary in order to compute the semiclassical imaginary part of the Minkowski effective action.

\section{CONCLUSIONS}

To conclude, the worldline instanton approach has now been extended to include also the quantum fluctuation prefactor for the nonperturbative imaginary part of the effective action. For general background fields the computation is numerical. Given the numerically determined instanton loop, the fluctuation determinant can be computed directly using (2.14), which is a result from the semiclassical analysis of quantum mechanical path integrals [41, 42]. For inhomogeneous time-dependent electric fields, the analysis can be done in much more explicit form, culminating in the semiclassical expression (3.58), which is expressed entirely in terms of the function $g\left(\gamma^{2}\right)$ defined in (3.44). Similarly, for inhomogeneous space-dependent electric fields, the corresponding expression is (4.4), with $\tilde{g}\left(\tilde{\gamma}^{2}\right)$ defined in (4.5). The agreement with Popov's WKB analysis [34] is perfect, and the semiclassical results match the numerical results of [39] very well. Within the semiclassical approximation it appears that the existence of a worldline instanton (i.e., the existence of a periodic solution to the classical Euclidean equations of motion) is a signal for the existence of an imaginary part to the Minkowski effective action, and hence for particle production. Since we are working in the semiclassical approximation we cannot necessarily conclude that, conversely, the absence of a worldline instanton solution would imply the absence of pair creation. Nevertheless, it is interesting to observe that, in the case of the spatially inhomogeneous electric field $E\left(x_{3}\right)=E \operatorname{sech}^{2}\left(k x_{3}\right)$ treated in section IV, there is no periodic worldline instanton 
when $\tilde{\gamma}>1$, and this is precisely the regime in which the imaginary part of the effective action vanishes, even away from the weak field limit [39, 49]. Similarly, it is easy to see from the classical equations of motion (2.6) that there is no worldline instanton for a plane-wave background field, consistent with the absence of pair production in this case [20]. A deeper physical and geometrical understanding of this correspondence would be interesting.

A number of important issues remain. First, while the agreement between our final answer and the worldline numerical approach are very good, the details of the calculation are very different. In this computation the nonperturbative result comes from small quantum fluctuations around a single closed loop amongst the ensemble of all closed loops. On the other hand, the worldline numerics does not appear to be dominated by single loops. For the electric field configurations considered here, the dominance of worldline instantons could directly be tested, for instance, by reweighting the numerical worldline ensemble with the instanton configurations. In turn, a cooling procedure on top of the worldline numerical algorithm can be used to numerically determine the instanton configurations needed for the present approach if applied to more complicated background fields. A better understanding of this correspondence should lead to more efficient numerical worldline loop computations, and should also clarify the physical nature of the semiclassical approximation for more general types of background field. This relates to the fact that the standard WKB approaches of Brézin and Itzykson [33], Popov et al [34], and Kim and Page [36], are difficult to generalize to more complicated fields as multidimensional WKB is considerably more difficult than one-dimensional WKB. On the other hand, the worldline fluctuation problem is inherently one-dimensional, once the worldline instanton loop has been found.

Second, the results here are for scalar QED. The generalization to spinor QED has been explained in [1] for the cases where the electric background is a function of just one spacetime coordinate, in which case the spin factor reduces to a factor of $-2(-1)^{n}$ inside the sum over multi-instantons of instanton number $n$. So for the leading single-instanton piece, the modification is simply the spin degeneracy factor of 2. However, for more complicated background fields, it is not clear how to evaluate the spin factor efficiently. This will be addressed in future work. Third, this paper has considered QED. The worldline expression (2.1) can be generalized also to nonabelian gauge theories [10, 15]. In this case the semiclassical worldline loops would then be related to Wong's equations [51]. This, in turn, may be useful for applications to the color glass condensate [52]. Finally, one could use the 
semiclassical approximation to address higher loop effects, using the higher loop worldline formalism for effective actions [10]. Thus the worldline instanton approach has the potential to address higher loops, while it is not at all clear how to address higher loops in the WKB language. The main result of Affleck et al's work [32] is that for a constant $E$ field, the instanton approach provides a way to resum the leading effect of all higher loops in the situation where the constant field $E$ is weak, but the coupling $e$ is arbitrary. This is because the instanton solution remains a stationary point even after taking the additional interaction term into account which in the worldline formalism represents virtual photon exchanges in the loop. It would be very interesting to try to extend this type of analysis to the general worldline instanton loops for inhomogeneous background fields. This should make contact with the work of Halpern et al, who considered a new type of strong-coupling expansion in the worldline approach [5].

Acknowledgements: We are very grateful to Don Page for helpful comments and correspondence. GD and QW thank the US DOE for support through the grant DE-FG0292ER40716. GD, CS and QW acknowledge the support of the NSF US-Mexico Collaborative Research Grant 0122615. HG acknowledges support by the DFG under contract Gi 328/1-3 (Emmy-Noether program) and Gi 328/3-2.

[1] G. V. Dunne and C. Schubert, "Worldline instantons and pair production in inhomogeneous fields," Phys. Rev. D 72, 105004 (2005) arXiv:hep-th/0507174.

[2] R. P. Feynman, "Mathematical formulation of the quantum theory of electromagnetic interaction", Phys. Rev. 80 440, (1950); "An Operator Calculus Having Applications in Quantum Electrodynamics", Phys. Rev. 84 108, (1951).

[3] J. L. Gervais and A. Neveu, "Feynman Rules for Massive Gauge Fields with Dual Diagram Topology", Nucl. Phys. B46 (1972) 381.

[4] Z. Bern, D. C. Dunbar, and T. Shimada, "String based methods in perturbative gravity", Phys. Lett. B 312 (1993) 277 arXiv:hep-th/9307001.

[5] M. B. Halpern, A. Jevicki and P. Senjanovic, "Field Theories in Terms of Particle-String Variables: Spin, Internal Symmetry and Arbitrary Dimension” , Phys. Rev. D 16, 2476 (1977); 
M. B. Halpern and W. Siegel, "The Particle Limit of Field Theory: A New Strong Coupling Expansion", Phys. Rev. D 16, 2486 (1977).

[6] A.M. Polyakov, "Gauge Fields and Strings", (Harwood Publ., 1987).

[7] R. R. Metsaev and A. A. Tseytlin, "On Loop Corrections To String Theory Effective Actions," Nucl. Phys. B 298, 109 (1988).

[8] Z. Bern and D. A. Kosower, "The Computation of loop amplitudes in gauge theories," Nucl. Phys. B 379, 451 (1992).

[9] M. J. Strassler, "Field theory without Feynman diagrams: One loop effective actions," Nucl. Phys. B 385, 145 (1992) arXiv:hep-ph/9205205.

[10] M.G. Schmidt and C. Schubert, "On the calculation of effective actions by string methods"; Phys. Lett. B 318 (1993) 438 hep-th/9309055; "Worldline Green functions for multiloop diagrams," Phys. Lett. B 331, 69 (1994) arXiv:hep-th/9403158; "Multiloop calculations in the string inspired formalism: The Single spinor loop in QED," Phys. Rev. D 53, 2150 (1996) arXiv:hep-th/9410100.

[11] M. Mondragon, L. Nellen, M. G. Schmidt and C. Schubert, "Yukawa couplings for the spinning particle and the worldline formalism," Phys. Lett. B 351, 200 (1995) arXiv:hep-th/9502125.

[12] E. D' Hoker and D. G. Gagné, "Worldline Path Integrals for Fermions with General Couplings", Nucl. Phys. B 467 (1996) 297 arXiv:hep-th/9512080.

[13] H. Kleinert and A. Chervyakov, "Reparametrization invariance of path integrals", Phys. Lett. B 464 (1999), 257 arXiv:hep-th/9906156.

[14] F. Bastianelli, O. Corradini, and P. van Nieuwenhuizen, "Dimensional regularization of the path integral in curved space on an infinite time interval", Phys. Lett. B 490 (2000) 154 arXiv:hep-th/0007105; F. Bastianelli and A. Zirotti, "Worldline formalism in a gravitational background", Nucl. Phys. 642 (2002) 372 arXiv:hep-th/0205182

[15] C. Schubert, "Perturbative quantum field theory in the string-inspired formalism," Phys. Rept. 355, 73 (2001) arXiv:hep-th/0101036.

[16] M. Reuter, M.G. Schmidt, and C. Schubert, "Constant external fields in gauge theory and the spin 0, 1/2, 1 path integrals", Ann. Phys. (N.Y.) 259 (1997) 313 hep-th/9610191.

[17] D. Cangemi, E. D'Hoker, and G. Dunne, "Derivative expansion of the effective action and vacuum instability for QED in (2+1) dimensions", Phys. Rev. D 51 (1995) 2513 arXiv:hep-th/9409113; V. P. Gusynin, I. A. Shovkovy, "Derivative expansion for the one 
loop effective Lagrangian in QED”, Can. J. Phys. 74 (1996) 282 arXiv:hep-ph/9509383.

[18] H.-T. Sato and M. G. Schmidt, "Worldline approach to the Bern-Kosower formalism two loop Yang Mills theory", Nucl. Phys. B 560 (1999) 551 arXiv:hep-th/9812229; H.-T. Sato, M.G. Schmidt, and C. Zahlten, "Two loop Yang-Mills theory in the worldline formalism and an Euler-Heisenberg type action", Nucl. Phys. B 579 (2000) 492 arXiv:hep-th/0003070.

[19] W. Heisenberg and H. Euler, "Consequences of Dirac's Theory of Positrons", Z. Phys. 98, 714 (1936).

[20] J. Schwinger, "On gauge invariance and vacuum polarization", Phys. Rev. 82, 664 (1951).

[21] W. Greiner, B. Müller and J. Rafelski, Quantum Electrodynamics Of Strong Fields, (Springer, Berlin, 1985).

[22] A. Casher, H. Neuberger and S. Nussinov, "Chromoelectric Flux Tube Model Of Particle Production," Phys. Rev. D 20, 179 (1979).

[23] Y. Kluger, E. Mottola and J. M. Eisenberg, "The quantum Vlasov equation and its Markov limit," Phys. Rev. D 58, 125015 (1998) arXiv:hep-ph/9803372.

[24] A. Ringwald, "Pair production from vacuum at the focus of an X-ray free electron laser," Phys. Lett. B 510, 107 (2001) arXiv:hep-ph/0103185; "Fundamental physics at an X-ray free electron laser", in Proceedings of Erice Workshop On Electromagnetic Probes Of Fundamental Physics, W. Marciano and S. White (Eds.), (River Edge, World Scientific, 2003) arXiv:hep-ph/0112254.

[25] S. M. Schmidt, D. Blaschke, G. Ropke, S. A. Smolyansky, A. V. Prozorkevich and V. D. Toneev, "A quantum kinetic equation for particle production in the Schwinger mechanism," Int. J. Mod. Phys. E 7, 709 (1998) arXiv:hep-ph/9809227; R. Alkofer, M. B. Hecht, C. D. Roberts, S. M. Schmidt and D. V. Vinnik, "Pair Creation and an X-ray Free Electron Laser," Phys. Rev. Lett. 87, 193902 (2001) arXiv:nucl-th/0108046; C. D. Roberts, S. M. Schmidt and D. V. Vinnik, "Quantum effects with an X-ray free electron laser," Phys. Rev. Lett. 89, 153901 (2002) arXiv:nucl-th/0206004.

[26] D. Kharzeev and K. Tuchin, "From color glass condensate to quark gluon plasma through the event horizon," Nucl. Phys. A 753, 316 (2005) arXiv:hep-ph/0501234.

[27] G. C. Nayak and P. van Nieuwenhuizen, "Soft-gluon production due to a gluon loop in a constant chromo-electric background field," Phys. Rev. D 71, 125001 (2005) arXiv:hep-ph/0504070; G. C. Nayak, "Non-perturbative quark-antiquark production from 
a constant chromo-electric field via the Schwinger mechanism," Phys. Rev. D 72, 125010 (2005) arXiv:hep-ph/0510052.

[28] W. Dittrich and H. Gies, "Probing the quantum vacuum. Perturbative effective action approach in quantum electrodynamics and its application," Springer Tracts Mod. Phys. 166, 1 (2000).

[29] T. Heinzl, B. Liesfeld, K. U. Amthor, H. Schwoerer, R. Sauerbrey and A. Wipf, "On the observation of vacuum birefringence," arXiv:hep-ph/0601076.

[30] A. Di Piazza, K. Z. Hatsagortsyan and C. H. Keitel, "Light diffraction by a strong standing electromagnetic wave," arXiv:hep-ph/0602039.

[31] E. Zavattini et al. [PVLAS Collaboration], "Experimental observation of optical rotation generated in vacuum by a magnetic field," arXiv:hep-ex/0507107.

[32] I. K. Affleck, O. Alvarez and N. S. Manton, "Pair Production At Strong Coupling In Weak External Fields," Nucl. Phys. B 197, 509 (1982).

[33] E. Brezin and C. Itzykson, "Pair Production In Vacuum By An Alternating Field," Phys. Rev. D 2, 1191 (1970);

[34] V. S. Popov, "Pair Production in a Variable External Field (Quasiclassical approximation)", Sov. Phys. JETP 34, 709 (1972), "Pair production in a variable and homogeneous electric field as an oscillator problem", Sov. Phys. JETP 35, 659 (1972); V. S. Popov and M. S. Marinov, "E+ E- Pair Production In Variable Electric Field," Yad. Fiz. 16, 809 (1972), [Sov. J. Nucl. Phys. 16 (1973), 449]; "Pair production in an Electromagnetic Field (Case of Arbitrary Spin)", Sov. J. Nucl. Phys. 15, 702 (1972); "Electron - Positron Pair Creation From Vacuum Induced By Variable Electric Field," Fortsch. Phys. 25, 373 (1977).

[35] L. V. Keldysh, "Ionization in the field of a strong electromagnetic wave", Sov. Phys. JETP 20, 1307 (1965).

[36] S. P. Kim and D. N. Page, "Schwinger pair production via instantons in a strong electric field," Phys. Rev. D 65, 105002 (2002) arXiv:hep-th/0005078; "Schwinger pair production in electric and magnetic fields," Phys. Rev. D 73, 065020 (2006) arXiv:hep-th/0301132.

[37] H. Gies and K. Langfeld, "Quantum diffusion of magnetic fields in a numerical worldline approach," Nucl. Phys. B 613, 353 (2001) arXiv:hep-ph/0102185; "Loops and loop clouds: A numerical approach to the worldline formalism in QED," Int. J. Mod. Phys. A 17, 966 (2002) arXiv:hep-ph/0112198. 
[38] M. G. Schmidt and I. O. Stamatescu, "Matter Determinants In Background Fields Using Random Walk World Line Loops On The Lattice," Mod. Phys. Lett. A 18, 1499 (2003); "Determinant calculations using random walk worldline loops," Nucl. Phys. Proc. Suppl. 119, 1030 (2003) arXiv:hep-lat/0209120.

[39] H. Gies and K. Klingmüller, "Pair production in inhomogeneous fields," Phys. Rev. D 72, 065001 (2005) arXiv:hep-ph/0505099.

[40] H. Gies, J. Sanchez-Guillen and R. A. Vazquez, "Quantum effective actions from nonperturbative worldline dynamics," JHEP 0508, 067 (2005) arXiv:hep-th/0505275.

[41] S. Levit and U. Smilansky, "A New Approach To Gaussian Path Integrals And The Evaluation Of The Semiclassical Propagator," Annals Phys. 103, 198 (1977).

[42] H. Kleinert, Path Integrals in Quantum Mechanics, Statistics, Polymer Physics, and Financial Markets, (World Scientific, Singapore, 2004).

[43] D. G. C. McKeon and T. N. Sherry, "Radiative effects in a constant magnetic field using the quantum mechanical path integral", Mod. Phys. Lett. A9 (1994) 2167.

[44] D. Fliegner, P. Haberl, M. G. Schmidt and C. Schubert, "The higher derivative expansion of the effective action by the string inspired method. II," Annals Phys. 264, 51 (1998) arXiv:hep-th/9707189.

[45] M. Morse, The Calculus of Variations In the Large, AMS Colloquium Series XVIII, (AMS, New York, 1934).

[46] M. Abramowitz and I. Stegun, Handbook of Mathematical Functions, (Dover, New York, 1972).

[47] J. B. Formiga and C. Romero, "On the differential geometry of curves in Minkowski space," arXiv:gr-qc/0601002

[48] G. V. Dunne and T. Hall, "On the QED effective action in time dependent electric backgrounds," Phys. Rev. D 58, 105022 (1998) arXiv:hep-th/9807031; "Borel summation of the derivative expansion and effective actions," Phys. Rev. D 60, 065002 (1999) arXiv:hep-th/9902064.

[49] A. I. Nikishov, "Barrier Scattering In Field Theory Removal Of Klein Paradox," Nucl. Phys. B 21, 346 (1970); N. B. Narozhnyi and A. I. Nikishov, "The simplest processes in a pairproducing field", Yad. Fiz. 11, 1072 (1970) [Sov. J. Nucl. Phys. 11 (1970) 596].

[50] S. P. Kim and D. N. Page, in preparation. We thank Don Page for communications on this comparison. 
[51] S. K. Wong, "Field And Particle Equations For The Classical Yang-Mills Field And Particles With Isotopic Spin," Nuovo Cim. A 65, 689 (1970).

[52] J. Jalilian-Marian, S. Jeon and R. Venugopalan, "Wong's equations and the small x effective action in QCD," Phys. Rev. D 63, 036004 (2001) arXiv:hep-ph/0003070; F. Gelis and R. Venugopalan, "Particle production in field theories coupled to strong external sources," arXiv:hep-ph/0601209. 